\title{
Avaliação do potencial de utilização da madeira de Schizolobium amazonicum "Paricá" e Cecropia hololeuca "Embaúba" para produção de painéis aglomerados
}

Setsuo IWAKIRI ${ }^{1}$, Florian ZELLER ${ }^{2}$, Juliana Afonso PINTO³, Maria Guadalupe Lomeli RAMIREZ ${ }^{4}$, Marina Moura SOUZA 5 , Rodrigo SEIXAS ${ }^{6}$

\section{RESUMO}

Esta pesquisa teve por objetivo avaliar o potencial de utilização de madeira de Schizolobium amazonicum (Paricá) e Cecropia hololeuca (Embaúba) para produção de painéis aglomerados. Foram produzidos painéis experimentais com densidade nominal de $0,70 \mathrm{~g} / \mathrm{cm}^{3}$, utilizando a resina uréia-formaldeído e partículas de madeira de Paricá e Embaúba, e mistura destas, em proporçôes de 75,50 e $25 \%$. A madeira de Pinus taeda foi utilizada como testemunha. Os painéis foram prensados com pressão específica de $40 \mathrm{kgf} / \mathrm{cm}^{2}$, temperatura de $160^{\circ} \mathrm{C}$ e tempo de prensagem de 8 minutos. Os resultados das avaliaçóes de propriedades de absorção de água, inchamento em espessura, ligação interna, módulo de elasticidade e módulo de ruptura, indicaram que as madeiras de Schizolobium amazonicum (Paricá) e Cecropia hololeuca (Embaúba) são tecnicamente viáveis para produção de painéis aglomerados.

PALAVRAS-ChaVE: Aglomerados, Paricá, Embaúba.

\section{Evaluation of potential use of the wood of Schizolobium amazonicum "Paricá" and Cecropia hololeuca "Embaúba" to particleboard manufacture}

\section{ABSTRACT}

This research was developed to evaluate the potential use of the woods Schizolobium amazonicum (Paricá) and Cecropia hololeuca (Embaúba) for manufacturing particleboard. Were made panels with the densities of $0.70 \mathrm{~g} / \mathrm{cm}^{3}$, using mixtures of urea-formaldehyde resin and wood particles of Paricá and Embaúba, in proportions of 75, 50 and 25\%. The Pinus taeda wood was used as the reference. The panels were pressed at the temperature of $160^{\circ} \mathrm{C}$, pressure of $40 \mathrm{kgf} / \mathrm{cm}^{2}$, for 8 minutes. The evaluations of the properties of water absorption, thickness swelling, internal bond, modulus of elasticity and modulus of ruptures, showed that the woods of Schizolobium amazonicum (Paricá) e Cecropia hololeuca (Embaúba) are technically feasible for particleboard manufacture.

KEYWORDS: Particleboard, Paricá, Embaúba.

\footnotetext{
1 Universidade Federal do Paraná, E-mail: setsuo@ufpr.br

2 Universidade Federal do Paraná, E-mail: f_zeller@gmx.net

${ }^{3}$ Universidade Federal do Paraná, E-mail: ju_sebaio@yahoo.com.br

${ }^{4}$ Universidade Federal do Paraná, E-mail: glomeli12@hotmail.com

${ }^{5}$ Universidade Federal do Paraná, E-mail: mourafloresta@yahoo.com.br

${ }^{6}$ Cikel Brasil Verde Madeiras Ltda, E-mail: rodrigoseixas@cikel.com.br
} 


\section{INTRODUÇÃO}

O setor de painéis de Madeira reconstituída no Brasil tem apresentado um grande crescimento a partir da década de 90 , com aumento expressivo na capacidade produtiva em função das implantações de novas unidades industriais de aglomerados e MDF. Grandes investimentos têm sido realizados também na implantação de novas áreas de plantios florestais para assegurar o suprimento de madeira requerido em grande escala pelas indústrias. De acordo com o estudo setorial do BNDES, a produção brasileira de painéis aglomerados cresceu de $866 \mathrm{mil} \mathrm{m}^{3} /$ ano em 1995 para 2,098 milhóes $\mathrm{de}^{3} \mathrm{em}$ 2005. O crescimento neste período foi de $9,3 \%$ ao ano, sendo um percentual bem superior ao $4,5 \%$ do nível mundial. A produção de painéis aglomerados no Brasil representa quase $46 \%$ do total de produçáo de painéis de madeira, incluindose os painéis compensados (BNDES, 2008). O grande crescimento do setor de painéis de madeira reconstituída pode ser atribuído à demanda crescente dos setores moveleiro e da construção civil.

As principais espécies utilizadas na produção de painéis aglomerados e MDF no Brasil são as dos gêneros Pinus e Eucalipto, as quais são plantadas em grande escala nas regiôes sul e sudeste do país, onde estão localizadas as indústrias. Nas regióes norte, nordeste e centro-oeste do país, além da ausência de pólos moveleiros que demandam grande quantidade de painéis, os plantios florestais em escala comercial ainda são restritos a pequenas áreas. Entretanto, a partir da década de 90, começaram a implantação de pequenas áreas de plantios de Schizolobium amazonicum (Paricá), visando o seu aproveitamento industrial, principalmente para produçáo de laminas para painéis compensados.

Os plantios em escala comercial de Paricá - Schizolobium amazonicum Huber ex. Ducke estão concentrados no Estado do Pará, especialmente na região de Paragominas. De acordo com dados da SBS (2006), estima-se que a área de plantios seja superior a 50 mil ha. A sua madeira apresenta coloraçấo branca e tem densidade média de $0,40 \mathrm{~g} / \mathrm{cm}^{3}$. O Paricá é uma espécie de madeira tropical que apresenta um crescimento acelerado, podem ser implantados em plantios homogêneos ou consorciados, e apresentam vantagens de ser resistente ao ataque de pragas e doenças. A sua produtividade média anual é na faixa de 20 a $30 \mathrm{~m}^{3} / \mathrm{ha} / \mathrm{ano}$, estando na mesma faixa de crescimento das espécies de Pinus que é de 25 a $30 \mathrm{~m}^{3} / \mathrm{ha} / \mathrm{ano}$, e superior a teca com 15 a $20 \mathrm{~m}^{3} / \mathrm{ha} / \mathrm{ano}$. A outra espécie com grande potencial para produção de aglomerados é a Cecropia hololeuca - Embaúba. Nas áreas de florestas nativas as árvores de Embaúba são eretas, sem ramificações, podendo atingir até $15 \mathrm{~m}$ de altura. A sua madeira é muito leve e esbranquiçada, com densidade em torno de $0,43 \mathrm{~g} / \mathrm{cm}^{3}$.

$\mathrm{Na}$ escolha de espécies de madeira para produçáo de painéis de madeira reconstituída devem ser considerados alguns parâmetros importantes como: densidade, $\mathrm{pH}$ e extrativos (Maloney, 1993; Tsoumis, 1993). A densidade da madeira é um dos requisitos básicos na escolha de espécies para produção de painéis aglomerados, por influenciar diretamente na sua razão de compactaçáo. Segundo os autores, a razão de compactaçáo é a relação entre a densidade do painel e densidade da madeira e, esta relaçấo, deve ser de no mínimo 1,3 para que ocorra uma densificação suficiente para a formação do painel. Kelly (1977), afirma que, para painéis de mesma densidade, produzidos com madeira de baixa densidade, as propriedades mecânicas serão superiores, entretanto, a sua estabilidade dimensional será inferior em comparação aos painéis produzidos com madeira de maior densidade. Segundo o autor, nos painéis com maior razáo de compactação, há maior quantidade de partículas de madeira e, consequentemente, ocorrerá maior densificação do painel, resultando em maior inchamento higroscópico da madeira e liberação das tensôes de compressão geradas durante o processo de prensagem.

A mistura de diferentes espécies na composiçâo do painel aglomerado é muito importante no sentido de ampliar a oferta de matéria-prima para estas indústrias que requerem grandes volumes de madeira. Diferentes características físicoquímicas das espécies podem ser consorciadas através da mistura homogênea das partículas de madeira para viabilizar o processo de colagem e formaçáo do painel. Moslemi (1974) apresenta uma fórmula para o cálculo da densidade média da madeira resultante da mistura de várias espécies com diferentes densidades:

$\mathrm{Dm}=\left[\mathrm{Dm}_{1} \mathrm{x}(\%)+\mathrm{Dm}_{2} \mathrm{x}(\%)+\ldots .+\mathrm{Dm}_{\mathrm{n}} \mathrm{x}(\%)\right]: \mathrm{n}$

Onde:

Dm: densidade da madeira resultante da mistura de espécies

$\mathrm{Dm}_{1,2, \ldots \mathrm{n}}:$ densidade da madeira das espécies

(\%): percentagem da espécie na mistura

Vários estudos têm sido realizados para avaliaçáo da mistura de espécies para produção de painéis aglomerados. Iwakiri et al. (1996) analisaram a influência da mistura de partículas de madeira de Pinus taeda com Eucalyputs dunnii nas proporçóes de 100, 75, 50, 25 e 0\% de Pinus em relação ao Eucalipto nas propriedades de painéis aglomerados experimentais. Os resultados indicaram a possibilidade de mistura de $E$. dunnii em proporçóes de até $50 \%$ com $P$. taeda sem prejudicar as propriedades físicas e mecânicas dos painéis aglomerados. Iwakiri et al. (1995) avaliaram também a influência da mistura de partículas de madeira de Pinus taeda com Mimosa scabrela (bracatinga) nas proporções de $100,75,50,25$ e $0 \%$ de pinus em relação à bracatinga sobre as propriedades de painéis aglomerados estruturais do tipo "waferboard". Os resultados apontaram para melhora 
na estabilidade dimensional dos painéis com o aumento na proporção de partículas de bracatinga na mistura com partículas de pinus.

Este trabalho teve como objetivo avaliar o potencial de utilização da madeira de Schizolobium amazonicum (Paricá) e Cecropia hololeuca (Embaúba) para produção de painéis aglomerados.

\section{MATERIAL E MÉTODOS}

\section{MATERIAL}

Foram utilizadas nesta pesquisa madeiras de Schizolobium amazonicum (Paricá), Cecropia hololeuca (Embaúba) e Pinus taeda, sendo esta, empregada como base referencial para comparaçôes de resultados, tendo em vista ser a espécie mais consumida na produçấo industrial de painéis aglomerados no Brasil.

As madeiras de paricá e embaúba foram coletadas na região de Paragominas, Estado do Pará, sendo a primeira, proveniente de plantios florestais e, a segunda, de uma área de floresta nativa. As toras foram transformadas em tábuas com uma polegada de espessura e transportadas até o local de estudos. A madeira de Pius taeda foi coletada na forma de partículas numa indústria de painéis aglomerados localizada no Município de Araucária, Estado do Paraná. Para a colagem de partículas foi utilizada a resina uréia-formaldeído com teor de sólidos de 65\%, pH 7,8 e viscosidade Brookfield de 450 cP.

\section{METODOLOGIA DE PESQUISA}

Os painéis aglomerados foram produzidos em escala laboratorial de acordo com o plano experimental apresentado na tabela 1 .

Tabela 1 - Plano experimental

\begin{tabular}{lcc}
\hline Tratamento & Espécie & Proporção de mistura \\
\hline T1 & Pinus & $100 \%$ \\
T2 & Paricá & $100 \%$ \\
T3 & Embaúba & $100 \%$ \\
T4 & Paricá / Embaúba & $75 \% / 25 \%$ \\
T5 & Paricá / Embaúba & $50 \% / 50 \%$ \\
T6 & Paricá / Embaúba & $25 \% / 75 \%$ \\
\hline
\end{tabular}

As partículas de madeira foram geradas num picador de disco na forma de "flakes" com as seguintes dimensôes nominais: comprimento de $25 \mathrm{~mm}$, espessura de $0,7 \mathrm{~mm}$ e largura variável. Após a secagem ao teor de umidade médio de $3 \%$, as partículas "flakes" foram reprocessadas no moinho de martelo e classificadas em peneira de malha 0,6 para retirada de finos.

A resina líquida após catalisada com sulfato de amônia foi aplicada sobre as partículas em quantidade de $8 \%$ de sólidos em relação ao peso seco das partículas. Para a formaçáo do colchão de partículas foi utilizada uma caixa formadora vazada com dimensóes laterais de 50 × $50 \mathrm{~cm}$. Os cálculos para definição de quantidade de materiais para formação de um painel foram feitos para densidade nominal de $0,75 \mathrm{~g} / \mathrm{cm}$ e dimensóes de 50 × 50 × $1,5 \mathrm{~cm}$. Os painéis foram prensados à temperatura de $160^{\circ} \mathrm{C}$, pressão específica de $40 \mathrm{kgf} / \mathrm{cm}^{2} \mathrm{e}$ tempo de prensagem de 8 minutos. Foram produzidos dois painéis por tratamento, perfazendo um total de doze painéis experimentais.

Após a prensagem, os painéis foram esquadrejados e acondicionados em câmara climática com temperatura de 20 $\pm 2^{\circ} \mathrm{C}$ e umidade relativa de $65 \pm 3 \%$, até sua estabilização ao teor de umidade médio de $12 \%$.

Para a avaliaçáo das propriedades físico-mecânicas dos painéis foram retirados de cada painel quatro corpos-de-prova para os ensaios de flexão estática visando determinação de módulo de elasticidade (MOE) e módulo de ruptura (MOR); cinco para ligação interna; e cinco para absorção de água e inchamento em espessura após 24 horas de imersão em água. Os ensaios foram realizados de acordo com os procedimentos descritos na Norma Européia EN 310, EN 319 e EN 317, respectivamente.

A análise estatística foi realizada segundo um delineamento inteiramente ao acaso e os resultados foram avaliados através da análise de variância e teste de Tukey ao nível de probabilidade de $95 \%$.

\section{RESULTADOS E DISCUSSÃO}

\section{RAZÃO DE COMPACTAÇÃO}

As densidades médias das espécies em estudo foram de $0,42 \mathrm{~g} / \mathrm{cm}^{3}, 0,32 \mathrm{~g} / \mathrm{cm}^{3}$ e $0,27 \mathrm{~g} / \mathrm{cm}^{3}$, respectivamente para madeiras de Pinus, Paricá e Embaúba. Na tabela 2 estão apresentadas as densidades médias da madeira após as mistura de espécies em diferentes proporçôes de Paricá e Embaúba, determinadas com base na fórmula apresentada por Moslemi (1974).

Para o tratamento T4 com $75 \%$ de Paricá e $25 \%$ de Embaúba, a densidade média da madeira foi de $0,307 \mathrm{~g} /$ $\mathrm{cm}^{3}$. Para as misturas em proporçôes de $50 \%$ x $50 \%$ e $25 \%$ x $75 \%$ de Paricá e Embaúba, as densidade médias foram respectivamente de $0,295 \mathrm{~g} / \mathrm{cm}^{3}$ e $0,282 \mathrm{~g} / \mathrm{cm}^{3}$.

Os valores de razão de compactação obtidos em relação à densidade do painel e densidade da madeira variaram de 1,609 a 2,429. Pode-se constatar que com o aumento na proporção da madeira de Embaúba em relação a madeira de Paricá (T4, T5 e T6), resultam em valores superiores de razão de compactação. Esta variação ocorre devido à menor densidade da madeira de Embaúba. Iwakiri et al. (1995) 
encontraram para painéis aglomerados produzidos com mistura de madeira de Pinus taeda com Eucalytus dunnii, valores de razão de compactação variando de 1,03 a 1,74 com o aumento na proporção de madeira de $P$. taeda em relação a madeira de. E. dunnii.

Tabela 2 - Razão de compactação dos painéis

\begin{tabular}{lccc}
\hline Tratamento & $\mathrm{DM}\left(\mathrm{g} / \mathrm{cm}^{3}\right)$ & $\mathrm{DP}\left(\mathrm{g} / \mathrm{cm}^{3}\right)$ & $\mathrm{RC}$ \\
\hline T1 - Pi 100 & 0,420 & 0,676 & 1,609 \\
T2 - Pa 100 & 0,320 & 0,687 & 2,146 \\
T3 - Em 100 & 0,270 & 0,684 & 2,533 \\
T4 - Pa 75 / Em 25 * & 0,307 & 0,680 & 2,214 \\
T5 - Pa 50 / Em 50 * & 0,295 & 0,687 & 2,328 \\
T6 - Pa 25/Em 75 * & 0,282 & 0,685 & 2,429 \\
\hline
\end{tabular}

Pi: pinus; Pa: paricá; Em: embaúba; DM: densidade da madeira $\left({ }^{*}\right.$ mistura de espécies); DP: densidade do painel; RC: razão de compactação.

\section{PROPRIEDADES FÍSICAS DOS PAINÉIS}

$\mathrm{Na}$ tabela 3 estáo apresentados os valores médios de absorção de água e inchamento em espessura após 24 horas de imersão em água dos painéis aglomerados.

Tabela 3 - Resultados de absorção de água e inchamento em espessura - 2 e 24 horas.

\begin{tabular}{lcc}
\hline Tratamento & $\mathrm{AA}-24 \mathrm{H}$ & $\mathrm{IE}-24 \mathrm{H}$ \\
\hline \multirow{2}{*}{ T1-Pi100 } & $90,66 \mathrm{~b}$ & $26,93 \mathrm{a}$ \\
& $(8,50)$ & $(9,36)$ \\
T2-Pa100 & $80,57 \mathrm{a}$ & $28,42 \mathrm{a}$ \\
& $(7,36)$ & $(8,25)$ \\
T3-Em100 & $79,22 \mathrm{a}$ & $26,19 \mathrm{a}$ \\
& $(7,58)$ & $(8,25)$ \\
T4-Pa75/Em25 & $82,22 \mathrm{ab}$ & $28,66 \mathrm{a}$ \\
& $(8,35)$ & $(7,65)$ \\
T5-Pa50/Em50 & $79,73 \mathrm{a}$ & $27,57 \mathrm{a}$ \\
& $(6,98)$ & $(6,84)$ \\
T6-Pa25/Em75 & $80,75 \mathrm{a}$ & $27,73 \mathrm{a}$ \\
& $(9,33)$ & $(8,25)$ \\
\hline
\end{tabular}

Pi: pinus; Pa: paricá; Em: embaúba; AA: Absorção de água; IE: inchamento em espessura; ( ... ) coeficiente de variação (\%).

Os resultados de absorção de água após 24 horas de imersão em água indicam para os painéis de Pinus, valor médio estatisticamente superior em comparação aos painéis de Paricá e Embaúba. Para os painéis produzidos com a mistura de madeiras de Paricá e Embaúba em diferentes proporçôes, não foram constatadas diferenças significativas entre as médias obtidas para esta propriedade. Pode-se constatar também que, a mistura de madeira de Embaúba em diferentes proporçôes com a madeira de Paricá, resultou em médias estatisticamente iguais em relaçấo aos painéis produzidos com $100 \%$ de Embaúba e 100\% de Paricá.

Os valores médios de absorção de água de $79,73 \%$ a $90,66 \%$, obtidos neste estudo, estấo compatíveis com os valores na faixa de $75,04 \%$ a $80,05 \%$, encontrados por Iwakiri et al. (1996), para painéis produzidos com mistura de madeiras de $P$. taeda e $E$. dunnii. Os valores um pouco superiores obtidos para o Paricá e Embaúba, podem ser atribuídos a menor densidade da madeira destas espécies, resultando em maiores quantidades de partículas de madeira na composição do painel, contribuindo para o aumento na área de absorção de água.

Com relação ao inchamento em espessura 24 horas, não foram constatadas diferenças estatisticamente significativas entre as médias dos tratamentos. Os valores médios foram muito próximos entre os tratamentos, variando de $26,19 \%$ para painéis de Embaúba (T3) e $28,66 \%$ para painéis produzidos com $75 \%$ de Paricá e $25 \%$ de Embaúba (T4).

Nos estudos realizados por Iwakiri et al. (1996) para painéis produzidos com mistura de madeiras de $P$. taeda e $E$. dunnii, os valores médios de inchamento em espessura variaram na faixa de 30,15\% 35,09\%. Embora os valores de razão de compactação dos painéis produzidos com madeiras de Paricá e Embaúba tenham sido bem superiores aos valores obtidos para os painéis de $P$. taeda e $E$. dunnii, as diferenças nos valores de inchamento em espessura náo foram táo significativas. Portanto, nâo pode ser comprovada para este caso, a influência da maior quantidade de partículas de madeira e liberação de maiores tensôes residuais de compressão sobre o inchamento em espessura dos painéis.

\section{PROPRIEDADES MECÂNICAS DOS PAINÉIS}

$\mathrm{Na}$ tabela 4 estão apresentados os valores médios obtidos para as propriedades de ligação interna, módulo de elasticidade e módulo de ruptura dos painéis aglomerados.

Tabela 4 - Resultados de ligação interna, módulo de elasticidade e módulo de ruptura.

\begin{tabular}{lccc}
\hline Tratamento & LI $(\mathrm{MPa})$ & $\mathrm{MOE}(\mathrm{MPa})$ & $\mathrm{MOR}(\mathrm{MPa})$ \\
\hline T1 - Pi 100 & $0,58 \mathrm{a}$ & $1.886,61 \mathrm{a}$ & $12,03 \mathrm{a}$ \\
& $(8,65)$ & $(7,65)$ & $(9,32)$ \\
T2 - Pa 100 & $0,76 \mathrm{bc}$ & $1.982,98 \mathrm{a}$ & $15,59 \mathrm{ab}$ \\
& $(6,59)$ & $(6,95)$ & $(7,36)$ \\
T3 - Em 100 & $0,68 \mathrm{ab}$ & $2.148,50 \mathrm{a}$ & $16,65 \mathrm{~b}$ \\
& $(9,32)$ & $(8,42)$ & $(8,35)$ \\
T4 - Pa 75 / Em 25 & $0,81 \mathrm{bc}$ & $2.012,53 \mathrm{a}$ & $16,44 \mathrm{~b}$ \\
& $(8,12)$ & $(9,32)$ & $(7,36)$ \\
T5 - Pa 50 / Em 50 & $0,88 \mathrm{C}$ & $2.081,72 \mathrm{a}$ & $16,29 \mathrm{~b}$ \\
& $(7,98)$ & $(8,78)$ & $(8,75)$ \\
& $0,88 \mathrm{C}$ & $2.006,86 \mathrm{a}$ & $15,02 \mathrm{ab}$ \\
T6 - Pa 25 / Em 75 & $(6,58)$ & $(6,35)$ & $(8,25)$ \\
\hline
\end{tabular}

Pi: pinus; Pa: paricá; Em: embaúba; LI: ligação interna; MOE: módulo de elasticidade; MOR: módulo de ruptura; ( ... ) coeficiente de variação (\%).

Os painéis produzidos com madeira de Paricá apresentaram valor médio de ligação interna estatisticamente superior em relação aos painéis de Pinus e igual aos painéis de Embaúba. Náo foi constatada diferença significativa entre as médias de ligação interna para os painéis de Pinus e de Embaúba. 
Para os painéis produzidos com diferentes proporçóes de mistura de Paricá e Embaúba não foram constatadas diferenças estatisticamente significativas entre os valores médios de ligação interna. Em termos de médias absolutas, as três diferentes proporçôes de mistura de Paricá e Embaúba resultaram em painéis com valores superiores de ligação interna em relação aos painéis produzidos com $100 \%$ de Pinus, Paricá e Embaúba. Os painéis produzidos com misturas Paricá x Embaúba de 50\% x 50\% (T5) e 25\% x 75\% (T6) apresentaram médias estatisticamente superiores em relação aos painéis com $100 \%$ de embaúba.

Os valores médios de ligação interna entre 0,68 a 0,88 MPa, obtidos para os painéis produzidos com $100 \%$ de Paricá e Embaúba, e com diferentes proporçôes de misturas, atendem ao requisito mínimo de $0,35 \mathrm{MPa}$, estabelecido pela Norma Européia EN 312-3: 1996.

Como parâmetros de referências apresentados na literatura, Iwakiri et al. (1996) encontraram para os painéis produzidos com mistura de madeiras de $P$. taeda com $E$. dunnii nas proporçôes de $75 \%$, 50\% e $25 \%$, valores médios de ligação interna na faixa de 0,87 a $0,91 \mathrm{MPa}$. Cabral et. al. (2007) encontraram para painéis produzidos com mistura de E. urophylla com P. elliottii e E. cloeziana com P. ellottii, valores médios de ligação interna de 0,45 e $0,56 \mathrm{MPa}$, respectivamente.

Com relação ao módulo de elasticidade (MOE), não foram constatadas diferenças estatisticamente significativas entre os valores médios obtidos para os painéis produzidos integralmente com as madeiras de Pinus, Paricá e Embaúba, e com a mistura destas espécies em diferentes proporçóes. Os valores médios de MOE variaram de 1.886,61 MPa a 2.148,50 $\mathrm{MPa}$, sendo o valor mínimo obtido para painéis de Pinus e o máximo para painéis de Embaúba. Estes valores atendem ao requisito mínimo de $1.600 \mathrm{MPa}$, estabelecido pela Norma Européia EN 312-3: 1996.

Os resultados de MOE referenciados na literatura são bastante abrangentes. Iwakiri et al. (1996) encontraram valores médios entre 2.208 a $2.530 \mathrm{MPa}$ para painéis produzidos com mistura de madeiras de $P$. taeda com E. dunnii, nas proporçóes de $75 \%, 50 \%$ e $25 \%$. Num outro estudo realizado por Iwakiri et. al. (2004) o valor médio de MOE obtido para painéis produzidos com madeira de Grevilea robusta foi de 1.463 MPa.

Os valores médios de módulo de ruptura (MOR) variaram de 12,03 MPa a 16,65 MPa, sendo o valor mínimo obtido para painéis de Pinus e o valor máximo para painéis de Embaúba. Com exceção do tratamento testemunha de Pinus, todos os demais painéis produzidos com madeiras de Paricá e Embaúba, e misturas destas espécies, atendem ao requisito mínimo de $13 \mathrm{MPa}$, estabelecido pela Norma Européia EN 312-3: 1996.
Os painéis produzidos com madeira de Embaúba apresentaram média estatisticamente igual em relação aos painéis de Paricá e superior em relação aos painéis de Pinus. Não houve diferença estatística entre os valores médios de MOR obtidos para os painéis de Paricá e Pinus. A mistura entre as madeiras de Paricá e Embaúba nas três diferentes proporçôes não resultaram em diferenças significativas entre os valores médios de MOR. As proporçôes de mistura de $75 \%$ e 50\% de Paricá em relaçẫo a Embaúba, resultaram em valores médios de MOR estatisticamente superiores em comparação aos painéis testemunhas de Pinus.

Os resultados de MOR obtidos neste estudo estáo compatíveis com os valores referenciados na literatura. Cabral et al. (2007) encontraram para painéis produzidos com misturas de madeira E. urophylla com P. elliottii e E. cloeziana com P. elliottii, valores de MOR de 16,7 MPa e 17,9 MPa, respectivamente. Iwakiri et al. (2004) obtiveram para painéis aglomerados de Grevilea robusta, valor médio de MOR de 10,3 MPa. Já, Batista et al. (2007) encontraram para painéis produzidos com madeira de $P$. elliottii e casca de E. pellita, valor de MOR de 5,0 MPa, considerado baixo em função da influência negativa da presença de casca na sua composição. Num outro estudo, Iwakiri et al. (1996) encontraram para painéis produzidos com mistura de $P$. taeda com $E$. dunnii, valores médios de MOR variando na faixa de $15,48 \mathrm{MPa}$ a $16,75 \mathrm{MPa}$.

\section{CONCLUSÕES E RECOMENDAÇÕES}

Com base nos resultados obtidos nesta pesquisa, as seguintes conclusões podem ser apresentadas:

- Os painéis produzidos com madeiras de Paricá e Embaúba apresentaram menor absorção de água em relação aos painéis testemunhas de Pinus. Não foram constatadas diferenças estatisticamente significativas nos valores de inchamento em espessura para todos os tratamentos avaliados.

- Os resultados de ligaçáo interna, módulo de elasticidade e módulo de ruptura dos painéis produzidos com madeiras de Paricá e Embaúba, e mistura destas, foram estatisticamente iguais ou superiores em relação aos painéis testemunhas produzidos com madeira de Pinus.

- O aumento na razão de compactação dos painéis aglomerados não influenciou de forma significativa nas suas propriedades físicas e mecânicas.

- Os resultados desta pesquisa indicam que as madeiras de Schizolobium amazonicum (Paricá) e Cecropia hololeuca (Embaúba) são tecnicamente viáveis para produção de painéis aglomerados. 


\section{AGRADECIMENTOS}

Os autores expressam seus agradecimentos às empresas Cikel Brasil Verde Madeiras Ltda e Hexion Química Indústria e Comércio Ltda, pelas doaçóes de madeiras e resina utilizadas nesta pesquisa.

\section{BIBLIOGRAFIA CITADA}

Batista, D.C.; Brito, E.O.; Setubal, V.G.; Góes, L.G. 2007. Threelayer particleboard production with Pinus elliottii Engelm wood and the addition of Eucalyptus pellita F. Muell bark. Cerne, 13(2): 178-187 (in Portuguese, with abstract in English).

BNDES. 2008. Technical Report. Wood panels in the Brazil: overview and perspectives. Rio de Janeiro. p. 121-156 (in Portuguese).

Cabral, C. P.; Vital, B. R.; Lucia, R. M. D.; Pimenta, A. S. 2007. Properties of particleboards manufactured with mixed particles from Eucalyptus spp and Pinus elliottii. Árvore, 31(5): 897-905 (in Portuguese, with abstract in English).

EUROPEAN COMMITTEE FOR STANDARDIZATION. Norma EN. 1993.

Iwakiri, S.; Klock, U.; Rocha, M.P.; Severo, E.T.D.; Rincoski,C.R.; Bortoletto, G.; Pio, N.S. 1995. Waferboard manufacture with species mixture. Agrárias, 14(1-2): 107-114 (in Portuguese, with abstract in English).

Iwakiri, S.; Latorraca, J.V.F.; Silva, D.A.; Gabardo, J.L.; Klitzke, R.J.; Fofano, A.; Fabrowski, F.; Interanmense. M.T. 1996. Particleboard manufacture from Pinus elliottii (Engelm) and Eucalyptus dunnii (Maid). Agrárias, 15(1): 33-41 (in Portuguese, with abstract in English).
Iwakiri S.; Shimizu, J.; Silva, J.C.; Del Menezzi, C.H.S; Puehringer, C.A.; Venson, I. Laroca, C. 2004. Particleboard manufacturing from Grevilea robusta A. Cunn. Ex. R. Br. Árvore, 28(6): 56-60 (in Portuguese, with abstract in English).

Kelly, M.W. 1977. Critical literature review of relationships between processing parameters and physical properties of particleboard. USDA For. Ser. Gen. Tech. Rep. FPL, Madison, USA. 66pp.

Maloney, T. M. 1993. Modern particleboard and dry-process fiberboard manufacturing. M. Freeman, San Francisco, USA. 689pp.

Moslemi, A.A. 1974. Particleboard vol. 1: Materials. Southern Illinois University Press, London. 244pp.

SBS, 2007. Facts and datas of the brazilian forestry - 2006. (www. sbs.org.br). Acesso: 12/01/2009 (in Portuguese).

Tsoumis, G. 1991. Science and technology of wood: structure, properties and utilization. Van Nostrand Reinhold, New York, USA. 494pp.

Recebido em 10/11/2008

Aceito em 03/11/2009 